\title{
Hirzebruch-Kato surfaces, Deligne-Mostow's construction, and new examples of negatively curved compact Kähler surfaces
}

\author{
FANGYANG ZHENG ${ }^{1}$
}

\begin{abstract}
In this article, we shall use the construction of Hirzebruch, Höfer, Kato, and Deligne-Mostow on compact complex 2-ball quotients to construct further finite Galois coverings over them, and by the analytical result of [M-S] and [Z], these coverings will admit Kähler metrics which are quasi-negatively curved, or negatively curved when the branching locus is smooth.
\end{abstract}

\section{Introduction.}

In this article, we continue our quest in [Z] for examples of quasi-negatively curved Kähler manifolds. Let $\mathcal{N}_{n}(n \geq 2)$ be the set of $n$-dimensional compact complex manifolds which admit Kähler metrics with quasi-negative sectional curvature (i.e., non-positive everywhere and negative somewhere).

It is generally believed that there should be abundant examples in each $\mathcal{N}_{n}$. However, due to the strong rigidity of such type of manifolds, very few examples are constructed so far. The first example of surface in $\mathcal{N}_{2}$ which is not biholomorphic to ball quotients was constructed in [M-S] in 1980. For $n>2$, such non-ball quotient example in $\mathcal{N}_{n}$ is still yet to be found.

The only successful attempt in finding such manifolds is by constructing finite branched covering over a compact ball quotient, with branching locus along a totally geodesic divisor ${ }^{2}$. The pull back of the canonical metric of the ball quotient gives a negatively curved metric on the covering, with

\footnotetext{
${ }^{1}$ Research partially supported by a NSF Grant and Alfred P. Sloan Fellowship. This project is also sponsored by the National Security Agency under grant \# MDA904-98-1-0036.

${ }^{2}$ In the real case, Gromov and Thurston [G-T] constructed lots of negatively curved compact (or complete with finite volume) Riemannian manifolds, by taking cyclic coverings over a compact real ball quotient along a totally geodesic submanifold of (real) codimension two.
} 
degeneration along the ramification locus. By adding a suitably chosen correction metric near the ramification divisor, one gets a genuine metric on the covering space which is quasi-negatively curved.

In [Z], we generalized the analytical result of [M-S] to higher dimensions and also relaxed the restriction on the totally geodesic divisor from smoothness to normal crossing. The further Fermat coverings of the three geometricly constructed ball quotients by Hirzebruch $([\mathrm{H}])$ then give three sequences of examples in $\mathcal{N}_{2}$, which are not ball quotients, and are different from Mostow-Siu's examples since they have different ratios of Chern numbers.

Since Hirzebruch's work, there have been more examples of smooth compact quotients of the complex 2-ball constructed by Höfer ([H1], [Ho]), Kato ([K2]), and the celebrated work of Deligne-Mostow [D-M]. (There was also the construction of Livné ([L]) on cyclic covers over the elliptic modular surface of level $N$, which also leads to some ball quotients). All of these ball quotients are finite Galois coverings over some relatively simple surfaces. The ramification divisors are necessarily totally geodesic with respect to the canonical metric on the ball quotients, since that metric is kept invariant by any automorphism.

The purpose of this article is to discuss further coverings over these geometricly constructed ball quotients. In the normal crossing case, the characterization theorem of Kato $[\mathrm{K}]$ allow us to get lots of such further coverings, which by the analytical result of [M-S] and [Z] (Theorem 2), are examples in $\mathcal{N}_{2}$. This demonstrates the abundance of surfaces in $\mathcal{N}_{2}$, which is our main goal in this article. See $\S 2$ for the detailed descriptions.

Theorem 11 in $\S 2.5$ can also be regarded as (a special case of) an uniqueness result for the Deligne-Mostow's construction. We verified the two dimensional cases (similar to Theorem 11), and we believe the higher dimensional cases should also hold ${ }^{3}$. Similar uniqueness phenomenon should also occur for the half integrality condition.

The paper is organized as following. In $\S 1$, we collect some results by Hirzebruch, Kato, and Deligne-Mostow. The results stated there are not in their full generality, instead they are adopted only to the extent and in the format to suit our later needs. In $\S 2$, we state our observations and examples.

\footnotetext{
${ }^{3}$ That is, if a smooth ball quotient $B^{n} / \Gamma$ can be a finite Galois cover over $Q_{s t}$ with branching locus away from $Q$, then $\Gamma$ should be commensurable with a Picard lattice. See $[\mathrm{D}-\mathrm{M}]$ or $\S 2.5$ for the notations.
} 
Acknowledgement. We would like to thank mathematicians C.K. Cheung, M. Davis, P. Deligne, G. Tian, H. Wu and S.T. Yau for helpful discussions. We would like to thank the referee for several valuable suggestions.

\section{Preliminaries.}

\subsection{Uniformization of smooth orbifolds.}

Let us first recall the definition of finite Galois coverings. Suppose $X^{n}$ is a compact ${ }^{4}$ complex manifold. A finite branched covering of $X$ is an irreducible normal complex analytic space $Y^{n}$ with a finite, surjective holomorphic map $f: Y^{n} \rightarrow X^{n}$ onto $X$. Denote by $R_{f}$ the subset of $Y$ where $f$ is not a local biholomorphism, and let $B_{f}=f\left(R_{f}\right)$ be its image. $R_{f}$ is called the ramification locus of the covering $f$, and $B_{f}$ the branching locus. Restrict $f$ to the open subset $f_{1}: Y \backslash f^{-1}\left(B_{f}\right) \rightarrow X \backslash B_{f}$, it becomes an unbranched finite covering. This corresponds to a subgroup of finite index $\pi_{1}\left(Y \backslash f^{-1}\left(B_{f}\right), y_{0}\right) \subseteq \pi_{1}\left(X \backslash B_{f}, x_{0}\right) . f$ is called Galois if this subgroup is normal. In this case, there is a finite deck transformation group $G \subseteq \operatorname{Aut}(Y)$ acting on $Y$, and $X=Y / G$ is the quotient. By a theorem of Grauert and Remmert ([G-R]), for any reduced, effective divisor $D$ in $X$, there is a one-one correspondence between the normal subgroups of finite index in $\pi_{1}\left(X \backslash D, x_{0}\right)$ and the finite Galois coverings over $X$ which are branched at most along $D$, i.e., with $B_{f} \subseteq D$.

In general, a finite Galois covering $Y$ over $X$ need not to be smooth. The following result of Kato ([K], Theorem 1 ) gives a necessary and sufficient condition for the smoothness of $Y$ in terms of the corresponding subgroup of $\pi_{1}\left(X \backslash D, x_{0}\right)$. In order to state his result, we need some terminologies first.

Let $U$ be a complex ${ }^{5}$ manifold and $b: U \rightarrow \mathbf{N}$ be a function into the set of positive integers. The pair $(U, b)$ is called uniformizable, if there is a connected complex manifold $Z$ and a discrete subgroup $\Gamma \subseteq A u t(Z)$ such that the quotient $Z / \Gamma$ is biholomorphic to $U$, and for any $z \in Z$, the order of the isotropy subgroup $\Gamma_{z}$ is equal to $b(\Gamma z)$. Here we identified $Z / \Gamma$ with $U$. In this case, $(Z, \Gamma)$ is called an uniformization of $(U, b)$.

In general, a pair $(X, b)$ of a complex manifold with an $\mathrm{N}$-valued function

\footnotetext{
${ }^{4}$ The compactness assumption is not necessary, we put is here simply because we only need this case in the article.

${ }^{5}$ Again this can be defined for the real case as well, but we will only be interested in the complex case.
} 
is called an orbifold ${ }^{6}$, if it is locally uniformizable, i.e., for any $x \in X$, there exits an open neighborhood $U$ of $x$ such that $\left(U,\left.b\right|_{U}\right)$ is uniformizable.

Example. Let $X$ be any complex manifold, and $D=\sum D_{i}$ be a normal crossing divisor in $X$. Arbitrarily assign an integer $b_{i} \geq 2$ to each $D_{i}$. Define $b: X \rightarrow \mathbf{N}$ by putting $b(x)$ equal to the product of $b_{i}$ for all the $D_{i}$ passing though $x$ (and let $b(x)=1$ if there is no such $\left.D_{i}\right)$. Then $(X, b)$ is an orbifold. We will call such an $(X, b)$ a normal crossing orbifold.

Let $(X, b)$ be a compact orbifold. Since $X \backslash b^{-1}(1)$ is a complex analytic subset, so we may denote by $D=\sum_{i=1}^{r} D_{i}$ its irreducible components of codimension 1 . For convenience, we will say the orbifold $(X, b)$ is of branching type $b=\left(b_{1}, \ldots, b_{r}\right)$, where $b_{i}=b(x)$ for a generic point $x \in D_{i}$.

Following Kato $([\mathrm{K}])$, let us denote by $H=\pi_{1}\left(X \backslash D, x_{0}\right)$, where $x_{0}$ is a generic point in $X$. (The base point will not be important in the following discussion). For each divisor $D_{i}$, let $\gamma_{i}$ be a small loop around $D_{i}$, (composed by a path from $x_{0}$ to a point on the loop and then come back). Let $H^{b}$ be the normal closure of $\left\{\gamma_{1}^{b_{1}}, \ldots, \gamma_{r}^{b_{r}}\right\}$ in $H$. For any $x \in X$, choose a small open ball $U$ centered at $x$ and define $H_{x}=\pi_{1}\left(U \backslash D, x_{0}\right)$ and $H_{x}^{b}$ similarly. Let $i_{x}: H_{x} \rightarrow H$ be the homomorphism induced by the inclusion map.

For any subgroup $K \subseteq H$, let $Z^{0}$ be the corresponding unbranched covering over $X \backslash D$. Let $Z$ be the Fox completion of $Z^{0}$ over $X$ ([F1]). It is a normal irreducible complex analytic space. We will call $Z$ the branched covering over $X$ corresponding to $K \subseteq H$.

Theorem (Kato). An orbifold $(X, b)$ is uniformizable if and only if for any $x \in X, i_{x}^{-1}\left(H^{b}\right)=H_{x}^{b}$. The corresponding covering is called the universal uniformization. More generally, the covering corresponding to a subgroup $K \subseteq H$ is an uniformization of $(X, b)$ if and only if for any $x \in X, i_{x}^{-1}(K)=$ $H_{x}^{b}$ holds. In this case, the universal uniformization factors through the one corresponding to $K$.

As an example, let us consider the one dimensional case. Let $\Sigma_{g}$ be a compact Riemann surface of genus $g$. Let $\left\{p_{1}, \ldots, p_{r}\right\}$ be $r$ distinct points and assign an integers $b_{i} \geq 2$ to each $p_{i}$. Then excluding the case $g=0, r=1$ and $g=0, r=2, b_{1} \neq b_{2}$, the orbifold is uniformizable. This is the classical solution of Fox to the Fenchel's conjecture ([F]). In the next subsection, we will see a (partial) generalization of this in the two dimensional case, the Hirzebruch-Kato surfaces.

\footnotetext{
${ }^{6} \mathrm{See}[\mathrm{T} 1]$ for the definitions and detailed discussions. In this article, we are only interested in the special case where the underlying space is smooth.
} 
In this article, we shall be mainly interested in the simplest situation. Following Hirzebruch $([\mathrm{H} 1])$, we define

Definition. Let $X^{n}$ be a compact complex manifold. A finite Galois covering $f: Y \rightarrow X$ is called a good covering, if $Y$ is smooth and the branching locus $B_{f}$ is a normal crossing divisor.

In other words, a good covering is just a finite uniformization over a normal crossing orbifold.

\subsection{Hirzebruch-Kato surfaces.}

In $[H]$, Hirzebruch constructed Fermat coverings of $\mathbf{P}^{2}$ branched along an arrangement of lines, and produced many interesting examples of algebraic surfaces. We refer the reader to $[\mathrm{H}]$ and $[\mathrm{H} 1]$ for details.

Let $L=\bigcup_{i=1}^{k} l_{i}$ be $k$ distinct lines in $\mathbf{P}^{2}$. Let $\Sigma=\left\{p_{k+1}, \ldots, p_{r}\right\}$ be the set of singular points, namely, those points where there are three or more lines in $L$ passing through.

We will always assume $\Sigma \neq \phi$, that is, $L$ is not in general position.

Let $X=X(L)$ be the blowing up of $\mathbf{P}^{2}$ at $\Sigma$. And denote by $D_{i}=\overline{l_{i}}$ $(1 \leq i \leq k)$ the proper transform of $l_{i}$ and $D_{\alpha}=E_{\alpha}(k+1 \leq \alpha \leq r)$ the exceptional divisor over $p_{\alpha}$, where $\Sigma=\left\{p_{k+1}, \ldots, p_{r}\right\}$.

Definition 1. A compact complex surface $Y$ is called a Hirzebruch-Kato surface, if it is a good covering over $X(L)$ for some line arrangement $L$ and has branching type $\left(b_{1}, \ldots, b_{r}\right)$, where $b_{i} \geq 2$ are arbitrary for $1 \leq i \leq k$, and $b_{\alpha}=m_{\alpha}(b, L)$ is defined to be the least common multiple of all the $b_{j}$ where $l_{j}$ passes through $p_{\alpha}$ and contains at least another singular point (if there is no such $l_{j}$ through $p_{\alpha}$, then set $\left.b_{\alpha}=1\right), k+1 \leq \alpha \leq r$.

We will call such a $Y$ a Hirzebruch-Kato surface of type $L^{b}=\sum_{i=1}^{k} b_{i} l_{i}$.

In [K1], it is proved that for any given $\left\{b_{1}, \ldots, b_{k}\right\}$, each $\geq 2$, and define $b_{\alpha}=m_{\alpha}(b, L)$ as above, the normal crossing orbifold $(X, b)$ admits a finite uniformization. That is, there will be Hirzebruch-Kato surfaces of type $L^{b}$. The construction is a direct generalization of the Fenchel-Fox theorem. We refer the readers to [K1] for the details.

In $\S 2$, we will see that any two Hirzebruch-Kato surfaces of the same type $L^{b}$ are commensurable, that is, they admit a common finite unbranched cover. So the specific subgroup of $\pi_{1}(X \backslash D)=\pi_{1}\left(\mathbf{P}^{2} \backslash L\right)$ will not be too important here. 
It would be very interesting to study the relationship between Hirzebruch-Kato surfaces over different arrangements, and the geography of their Chern numbers distribution. We believe that further exploration of this class will produce new and interesting examples of algebraic surfaces. Any higher dimensional generalizations would also be very interesting. It is worth noting that in $[\mathrm{Hu}]$, Hunt studied the interesting problem of threefold geography by considering the Fermat coverings over plane arrangements in $\mathbf{P}^{3}$. A generalization of Hirzebruch-Kato covering to three dimension will provide an even richer class of algebraic threefolds.

Example: Fermat coverings. In $[\mathrm{H}]$, Hirzebruch constructed Fermat coverings over any arrangement $L$, they are good coverings over $X(L)$ of constant branching type: $b_{i}=n$ for all $1 \leq i \leq r . n$ is called the order of the Fermat covering. Among them, he found three arrangements which, for suitable $n$, the Fermat covering becomes quotient of the unit ball $B^{2}$ in $\mathbf{C}^{2}$.

Example: Homogeneous coverings. In [Ho] and [H1], more ball quotients were found, they are good coverings over $X(L)$ with the following type:

$$
b_{i}=n, 1 \leq i \leq k, \quad \text { and } \quad b_{\alpha}=m, k+1 \leq \alpha \leq r
$$

where $n$ and $m$ are positive integers. Note that unlike the Fermat covering case, where the good coverings always exist, for a given $L$ and given $(n, m)$, there may not be any good coverings over $X(L)$ with the homogeneous type $b_{i}=n, b_{\alpha}=m$.

In [H1] (p.140), a complete list of all possible $(n, m)$ over the so-called homogeneous arrangements is given for the corresponding coverings to be ball quotient. In these cases, the existence of the coverings was obtained by Höfer in [Ho]. Over the complete quadrilateral, there are four homogeneous coverings which are ball quotients. They are part of the Deligne-Mostow's list (see the next subsection), since we will see in $\S 2.1$ that any two good coverings of the same branching type will be commensurable.

\subsection{Deligne-Mostow's construction.}

In [D-M], Deligne and Mostow used the monodromy map of the hypergeometric functions to produce lattices in $P U(1, n), n \leq 5^{7}$. In the two

\footnotetext{
${ }^{7}$ The program was started by Picard a hundred years ago, and was also studied by Terada $[\mathrm{Te}]$. See $[\mathrm{D}-\mathrm{M}]$ for the history and references.
} 
dimensional case, their result can be stated as the following.

Write $X_{I}=X(L)$, where $L$ is the complete quadrilateral arrangement:

$$
L=\left\{\left(z_{0}: z_{1}: z_{2}\right) \in \mathbf{P}^{2} \mid z_{0} z_{1} z_{2}\left(z_{0}-z_{1}\right)\left(z_{1}-z_{2}\right)\left(z_{2}-z_{0}\right)=0\right\}
$$

$X_{I}$ is the blowing up of $\mathbf{P}^{2}$ at the four triple points. The resulting ten curves $D=\bigcup_{i=1}^{6} \overline{l_{i}} \cup \bigcup_{\alpha=7}^{10} E_{\alpha}$ are all $(-1)$ curves, and there is an unique way to label them as $D_{i j}$ with $0 \leq i<j \leq 4$ such that $D_{i j} \cap D_{k l} \neq \phi \Leftrightarrow\{i, j\} \cap\{k, l\}=\phi$.

$Q=X_{I} \backslash D$ is the moduli spaces of 5-punctured $\mathbf{P}^{1}$. Let $\mu=\left(\mu_{0}, \ldots, \mu_{4}\right)$ be any 5 -uple of numbers such that $0<\mu_{i}<1$ for each $i, \sum_{i=0}^{4} \mu_{i}=2$, and satisfies the integrality condition (INT):

$$
\left(1-\mu_{i}-\mu_{j}\right)^{-1} \in \mathbf{Z}, \quad \forall 0 \leq i<j \leq 4 \text { with } \mu_{i}+\mu_{j}<1 .
$$

Then the image of the monodromy map $\rho: \pi_{1}(Q) \rightarrow P U(1,2)$ will be a lattice, called a Picard lattice. For any sublattice $\Gamma_{0} \subseteq \Gamma$ which acts freely on $B^{2}$, the normal subgroup of finite index $\rho^{-1}\left(\Gamma_{0}\right) \subseteq \pi_{1}(Q)$ realizes the smooth quotient of the ball, $B^{2} / \Gamma_{0}$, as a finite Galois covering over $Q_{s t}$, the stable extension of $Q$ with respect to $\mu$, and the branching locus is contained in $Q_{s t} \backslash Q$. We will call such a ball quotient $B^{2} / \Gamma_{0}$ a Deligne-Mostow quotient.

For five punctures, there are 27 solutions of $\mu$ which satisfies the integrality condition (INT). In 8 cases among those $27, Q_{s t}=X_{I}$. In these eight cases, $Q_{s t} \backslash Q=D$ is normal crossing, so a Deligne-Mostow quotient $Y=B^{2} / \Gamma_{0}$ is a finite uniformization of $\left(X_{I}, b\right)$, with

$$
b_{i j}=\left(1-\mu_{i}-\mu_{j}\right)^{-1} \text { along } D_{i j}
$$

These eight solutions of $\mu=\left(\mu_{0}, \ldots, \mu_{4}\right)$ are

$$
\begin{aligned}
\text { 1. } & \mu & =\frac{1}{5}(2,2,2,2,2) \\
\text { 2. } & \mu & =\frac{1}{8}(4,3,3,3,3) \\
\text { 3. } & \mu & =\frac{1}{9}(2,4,4,4,4) \\
\text { 4. } & \mu & =\frac{1}{12}(4,5,5,5,5) \\
\text { 5. } & \mu & =\frac{1}{12}(6,5,5,4,4) \\
\text { 6. } & \mu & =\frac{1}{12}(6,3,5,5,5) \\
\text { 7. } & \mu & =\frac{1}{15}(8,4,6,6,6)
\end{aligned}
$$




$$
\text { 8. } \quad \mu=\frac{1}{24}(14,7,9,9,9)
$$

Note that the first one gives the Fermat covering of order 5 , the next three are homogeneous coverings of type $(n, m)$ (that is, four disjoint lines will have $b_{\alpha}=m$ and the rest six lines have $\left.b_{i}=n\right)$ equal to $(4,8),(9,3)$, and $(6,4)$, respectively. The seventh one gives $3,3,3,5,5,5 ; 15,15,15,5$, which is a Hirzebruch-Kato surface. The first and the seventh are the only HirzebruchKato surfaces among these eight types.

We will see in $\S 2.5$ that, up to commensurability, these eight are the only smooth quotients of the ball that can be a good cover over $X_{I}$, with branching locus contained in $D$.

\section{Construction of quasi-negatively curved surfaces.}

\subsection{Good coverings.}

Throughout this subsection, we shall always assume that $X^{n}$ is a compact complex manifold, and $D=\sum_{i=1}^{r} D_{i}$ a normal crossing divisor in $X$. Also fix a point $x_{0} \in X \backslash D$ and write $H=\pi_{1}\left(X \backslash D, x_{0}\right)$. Let $\gamma_{i}$ be a small loop around $D_{i}$. For a $r$-uple of positive integers $b=\left(b_{1}, \ldots, b_{r}\right)$ and any $x \in X$, define $H^{b}, H_{x}^{b}$, and $i_{x}: H_{x} \rightarrow H$ as in $\S 1.1$. Note that here we allow $b_{i}$ to be equal to 1 .

Define $\mathcal{K}_{X, D}$ to be the set of all normal subgroups $K \subseteq H$ of finite index which are $b$-complete for some positive integer $r$-uple $b$, i.e., for any $x \in X$, $i_{x}^{-1}(K)=H_{x}^{b}$.

By Kato's theorem, good coverings over $X$ with branching locus contained in $D$ are in one-one correspondence with subgroups in $\mathcal{K}_{X, D}$. We will call the corresponding $r$-uple $b=\left(b_{1}, \ldots, b_{r}\right)$ the branching type of a good covering $Y$ or subgroup $K . b_{i}$ is just the multiplicity of $f: Y \rightarrow X$ at any $f^{-1}(x)$ for a generic point $x \in D_{i}$ or equivalently, the order of the image of $\gamma_{i}$ in $H / K$. Of course $B_{f}$ is just the union of those $D_{i}$ with $b_{i} \geq 2$.

The following observation is a direct consequence of Kato's theorem, nevertheless it provides the starting point of our examples of (quasi-)negatively curved surfaces.

Proposition 1. Let $X^{n}$ and $D$ be as above. Let $K, K^{\prime}$ be in $\mathcal{K}_{X, D}$, with branching type $b, b^{\prime}$, respectively. Then $K \cap K^{\prime}$ is also in $\mathcal{K}_{X, D}$, and its branching type $b^{\prime \prime}$ is the least common multiple of $b$ and $b^{\prime}$, that is, $b_{i}^{\prime \prime}=\left[b_{i}, b_{i}^{\prime}\right]$ for each $i$. 
Proof. For any $x \in X$, we have

$$
i_{x}^{-1}\left(K \cap K^{\prime}\right)=i_{x}^{-1}(K) \cap i_{x}^{-1}\left(K^{\prime}\right)=H_{x}^{b} \cap H_{x}^{b^{\prime}}=H_{x}^{b^{\prime \prime}}
$$

The last equality holds because, under our assumption, $D$ is normal crossing, so there is always a neighborhood $U$ of $x$ such that $U \backslash D$ is biholomorphic to a product $\left(\Delta^{*}\right)^{p} \times \Delta^{n-p}$, where $\Delta$ and $\Delta^{*}$ are the unit disc and punctured unit disc in $\mathbf{C}$, respectively. So $H_{x}$ is free abelian.

The next is a simple consequence of assumptions that $D$ is normal crossing, $Y$ is smooth, and $f$ is Galois (so the deck transformation group acts transitively on each fiber of $f$ ).

Proposition 2. Let $f: Y \rightarrow X$ be a good covering with $B_{f} \subseteq D$. Then $R_{f}=f^{-1}\left(B_{f}\right), R_{f}$ is normal crossing, and for each $D_{i}$ in $B_{f}, R_{i} \equiv f^{-1}\left(D_{i}\right)$ is the disjoint union of isomorphic smooth divisors, each is a good cover over $D_{i}$.

Let $d$ be the degree of $f$. Then all the Chern numbers of $Y$ divided by $d$ can be computed by the data on $X, D$, and the branching type $b$ (that is, two coverings with the same $b$ will have equal Chern number ratios).

By a theorem of Yau $[\mathrm{Y}]$, if $c_{1}(Y)<0$, which is equivalent to that $K_{X}+\sum\left(1-\frac{1}{b_{i}}\right) D_{i}$ being ample, then $Y$ will be a quotient of the complex unit ball $B^{n}$ if and only if the first two Chern numbers of $Y$ satisfies the equality

$$
(-1)^{n}\left(n c_{1}^{n}-2(n+1) c_{1}^{n-2} c_{2}\right)=0
$$

(and in general, when $c_{1}(Y)<0$, the left hand side is non-positive). That gives an effective way to detect the ball quotients when $X$ and $D$ are explicitly given. By Tian-Yau's theorem (see $\S 2.2$ ), this characterization can be given on the orbifold $(X, b)$ even without knowing the existence of a finite uniformization. We will discuss this in the next subsection.

The last sentence in Proposition 2 can be proved directly by induction on the dimensions, or by using the following corollary of Proposition 1. But first let us introduce a terminology.

Definition. Two complex manifolds are called commensurable, if they have a common finite unbranched cover.

Proposition 3. Let $Y, Y^{\prime}$ and $Y^{\prime \prime}$ be the good coverings of $X$ corresponding to $K, K^{\prime}$ and $K^{\prime \prime}=K \cap K^{\prime}$. Then $Y^{\prime \prime}$ is a good cover over $Y$, with 
branching locus contained in $R_{f}=f^{-1}\left(B_{f}\right)$, where $f: Y \rightarrow X$. The branching multiplicity of $Y^{\prime \prime} \rightarrow Y$ along any component of $R_{i}=f^{-1}\left(D_{i}\right)$ is $b_{i}^{\prime \prime} / b_{i}$. Similarly, $Y^{\prime \prime} \rightarrow Y^{\prime}$ is also a good covering.

In particular, if $Y$ and $Y^{\prime}$ over $X$ have equal branching type $b=b^{\prime}$, then they are commensurable.

Now suppose we have good coverings $Y$ and $Y^{\prime}$ over $X$ such that $Y$ is a quotient of the complex ball and $b \neq b^{\prime \prime}$, where $b^{\prime \prime}=\left[b, b^{\prime}\right]$. Let $h$ be the canonical complex hyperbolic metric on $Y$. It is an invariant metric, that is, any biholomorphism of $Y$ is an isometry of $h$. So each component of $R_{f}$, being fixed by some element of the deck transformation group, becomes fixed point set of an $h$-isometry. So it is totally geodesic with respect to $h$. Therefore, $Y^{\prime \prime}$ is a good covering over the ball quotient $Y$ along a normal crossing, totally geodesic divisor $R$ contained in $R_{f}$. (Since $b \neq b^{\prime \prime}$, this is truly a branched covering). By the analytical result of [M-S] (Lemma 2) and [Z] (Theorem 2), we know that $Y^{\prime \prime}$ will admit a Kähler metric whose complex curvature operator is negative definite in the complement of the singularities of the ramification locus of $Y^{\prime \prime} \rightarrow Y$, which is a subvariety of codimension at least two. (In case the set $\sum_{b_{i}^{\prime \prime}>b_{i}} D_{i}$ is smooth, the complex curvature operator of $Y^{\prime \prime}$ is negative definite everywhere). In particular, these manifolds are all strongly rigid in the sense that, any compact Kähler manifold homotopy equivalent to such a manifold must also be biholomorphic or antibiholomorphic to it. This is the strong rigidity theorem of Siu, generalizing (in the complex case) the Mostow rigidity theorem for locally symmetric spaces.

To simplify our later discussions, let us introduce a couple of notations. By Proposition 1, all good coverings over $X$ with the same branching type $b$ are commensurable, so we shall simply concentrate on the branching type vectors $b$.

Suppose $X^{n}$ is compact complex manifold, and $D=\sum_{i=1}^{r} D_{i}$ is a normal crossing divisor in it. Let us fix the order of the divisors $D_{i}$ here. Consider the space $\mathbf{N}^{r}$ of $r$-uples of positive integers. Let

$$
\mathcal{G}_{X, D}=\left\{b \in \mathbf{N}^{r} \mid(X, b) \text { has a finite uniformization. }\right\}
$$

Here as before, $(X, b)$ means the normal crossing orbifold with $b(x)$ equal to the product of $b_{i}$ for all $D_{i}$ passing through $x$ ( 1 if none). Any two such finite uniformizations (or equivalently, good coverings with the same branching type) are commensurable to each other. For two elements $b$ and $b^{\prime}$ in $\mathcal{G}$, define the partial order

$$
b \preceq b^{\prime} \Leftrightarrow \quad b_{i} \mid b_{i}^{\prime} \forall i
$$


and $b \prec b^{\prime}$ if $b \preceq b^{\prime}$ and $b \neq b^{\prime}$.

For simplicity, we will also denote by $\left[b, b^{\prime}\right]$ the vector whose $i$-th component is the least common multiple $\left[b_{i}, b_{i}^{\prime}\right]$, and call it the least common multiple of $b$ and $b^{\prime}$. It is after both $b$ and $b^{\prime}$ in the above order.

Now define $\mathcal{B}_{X, D}$ to be the subset of $\mathcal{G}_{X, D}$ where the finite uniformization is a quotient of the complex unit ball, and let

$$
\mathcal{G}_{X, D}^{+}=\left\{b \in \mathcal{G} \mid b \succ b^{\prime} \text { for some } b^{\prime} \in \mathcal{B}\right\}
$$

Summerize the above discussions, we have

Theorem 4. Let $X^{n}$ be a compact Kähler manifold and $D=\sum_{i=1}^{r} D_{i}$ a normal crossing divisor in $X$. If $b \in \mathcal{G}^{+}$, and $Y$ is any good covering of $X$ with branching type $b$ along $D$, then a finite unbranched covering of $Y$ will admit a Kähler metric whose complex curvature operator is negative definite in the complement of a subvariety with codimension at least two.

Note that these manifolds are not ball quotients themselves ( $§ 2.3$, Proposition 7).

So the key here is to find examples where $\mathcal{B} \neq \phi$, and to have $b^{\prime}$ strictly after some element $b$ of $\mathcal{B}$.

\subsection{A theorem of Tian and Yau.}

In [T-Y], Tian and Yau generalized the work of Yau ([Y]) and Cheng-Yau ([C-Y], [C-Y1]) on the existence of Kähler-Einstein metrics, and give a complete characterization of the orbit spaces of a discrete subgroup with finite covolume acting on the complex unit ball. We will only need the special case when the quotient orbifold is compact and with smooth underlying space.

Let $X^{n}$ be a projective manifold, and $D=\sum_{i=1}^{r} D_{i}$ a normal crossing divisor in $X$. We fix the order of $D_{i}$ here. Let $b=\left(b_{1}, \ldots, b_{r}\right)$, where each $b_{i} \geq 2$ is an integer. Consider the normal crossing orbifold $(X, b)$. One can define the Chern classes for the orbifold, denoted by $\overline{c_{i}}$. We will only need the first two:

$$
\begin{aligned}
& \overline{c_{1}}=c_{1}(X)-\sum_{i=1}^{r}\left(1-\frac{1}{b_{i}}\right) D_{i} \\
& \overline{c_{2}}=c_{2}(X)+\sum_{i=1}^{r}\left(1-\frac{1}{b_{i}}\right)\left(K_{X} D_{i}+D_{i}^{2}\right)+\frac{1}{2} \sum_{i \neq j}\left(1-\frac{1}{b_{i}}\right)\left(1-\frac{1}{b_{j}}\right) D_{i} D_{j}
\end{aligned}
$$


Up to common positive multiples, these are just the Chern classes of a finite uniformization of $(X, b)$. To be more precise, if $Y$ is a finite uniformization of $(X, b)$ and let $d$ be the degree of $Y$ over $X$. Then

$$
c_{1}^{n}(Y) / d={\overline{c_{1}}}^{n}, \quad c_{1}^{n-2}(Y) c_{2}(Y) / d={\overline{c_{1}}}^{n-2} \overline{c_{2}}
$$

In [T-Y], Tian and Yau proved that if $-\overline{c_{1}}$ is ample, then there exists an unique Kähler-Einstein metric of negative Ricci curvature on $X$ with singularity along $D$ that is compatible with the orbifold structure of $(X, b)$, i.e., when lifted to a local uniformization, the metric becomes a non-singular (usual) metric. Furthermore, the inequality

$$
(-1)^{n}{\overline{c_{1}}}^{n} \leq(-1)^{n} \frac{2(n+1)}{n}{\overline{c_{1}}}^{n-2}{\overline{c_{2}}}
$$

holds, with equality if and only if the metric has constant holomorphic sectional curvature. In this case, $(X, b)$ is the quotient of the complex unit ball by a lattice $\Gamma$. Passing to torsion free sublattice, we get a finite uniformization $Y$ of $(X, b) . Y$ is a smooth compact quotient of the ball. In summary, a special case of Tian-Yau's theorem gives the following:

Theorem (Tian-Yau). Let $X^{n}$ be a projective manifold, and

$$
D=\sum_{i=1}^{r} D_{i}
$$

a normal crossing divisor in $X$. Let $b=\left(b_{1}, \ldots, b_{r}\right)$, where each $b_{i} \geq 2$ is an integer. Then $(X, b)$ admits a finite uniformization which is a ball quotient if and only if

$$
\overline{c_{1}}<0,{\overline{c_{1}}}^{n}=\frac{2(n+1)}{n}{\overline{c_{1}}}^{n-2}{\overline{c_{2}}}
$$

In other words, $b \in \mathcal{B}_{X, D}$ once the above two necessary numerical conditions are satisfied. This gives a powerful way to produce compact smooth ball quotients geometrically. We will see in $\S 2.7$ some new examples of 2-ball quotients.

In the next subsection, we will give the details of the orbifold Chern numbers computation in the case of dimension two. For higher dimensions, one can either use the virtual exact sequence in $[\mathrm{T}-\mathrm{Y}](\S 3)$, or by taking a sufficiently large integer $m$ and a smooth surface $S^{2} \subseteq X^{n}$ which is the intersection of $n-2$ generic members in the linear system $\left|-m \overline{c_{1}}\right|$, and 
then restrict everything down to $S$, and reduce it to the two dimensional situation ${ }^{8}$.

\subsection{Ratio of the Chern numbers .}

From now on we will concentrate on the two dimensional case, although the computations in this subsection can also be carried out in general dimensions as well. Let $f: Y^{2} \rightarrow X^{2}$ be a good covering of degree $d$ with branching locus $B_{f}=\sum_{i=1}^{r} D_{i}$. Write $R_{i}=f^{-1}\left(D_{i}\right)$, and $R_{f}=\sum_{i=1}^{r} R_{i}$. Each $R_{i}$ is a smooth (may be disconnected) curve, and $f$ has multiplicity $b_{i}$ along each component of $R_{i}$. The following computation of Chern numbers is standard, so we omit the proof.

Proposition 5. For a good covering $f: Y^{2} \rightarrow X^{2}$ of degree d with branching locus $B_{f}=\sum_{i=1}^{r} D_{i}$ and multiplicity $b_{i}$ along $R_{i}=f^{-1}\left(D_{i}\right)$, the Chern numbers of $Y$ are:

$$
\begin{aligned}
c_{1}^{2}(Y) / d= & c_{1}^{2}(X)+\sum_{i=1}^{r}\left[2\left(1-\frac{1}{b_{i}}\right) K_{X} D_{i}+\left(1-\frac{1}{b_{i}}\right)^{2} D_{i}^{2}\right] \\
& +\sum_{i \neq j}\left(1-\frac{1}{b_{i}}\right)\left(1-\frac{1}{b_{j}}\right) D_{i} D_{j} \\
c_{2}(Y) / d= & c_{2}(X)+\sum_{i=1}^{r}\left(1-\frac{1}{b_{i}}\right)\left(K_{X} D_{i}+D_{i}^{2}\right) \\
& +\frac{1}{2} \sum_{i \neq j}\left(1-\frac{1}{b_{i}}\right)\left(1-\frac{1}{b_{j}}\right) D_{i} D_{j}
\end{aligned}
$$

The next is the local version of Hirzebruch's proportionality principle, which is due to Enoki ([H1], p. 142), that we shall need later. This can be seen quite transparently from the differential geometric point of view, since for any local unitary frame $\left\{e_{i}\right\}$ in a ball quotient $Y^{n}$, the curvature of the canonical metric takes the simple form

$$
R_{i \bar{i} i \bar{i}}=-2 ; \quad R_{i \bar{i} j \bar{j}}=-1, i \neq j
$$

So when $C$ is a totally geodesic submanifold in $Y$, the curvature of $C$ (for the restriction metric) is just the restriction of the curvature of $Y$, and the

${ }^{8} \mathrm{M}$. Davis showed us a simple way to obtain $\overline{c_{i}}$ in a neat formula, by a nice local splitting principle $[\mathrm{D}]$. 
Chern forms can be expressed in terms of these curvatures. The advantage of a differential geometric argument like this is, it gives inequalities for general submanifolds, while equalities characterize the totally geodesy, and it can also work for the (finite volume) non-compact complete case. (See [Z], §3 for more details). In this paper, we just need the simplest case:

Proposition 6 (Hirzebruch-Enoki local proportionality). If $Y^{2}$ is a compact smooth quotient of the complex 2-ball and $C$ is a smooth, totally geodesic curve (may be a disjoint union), then $\left(K_{Y}+3 C\right) C=0$.

Now let us come back to our good coverings. For a given surface $X^{2}$ and normal crossing divisor $D=\sum_{i=1}^{r} D_{i}$ in $X$ (here again we fix the order of $D_{i}$ ), our goal is to find examples in $\mathcal{G}_{X, D}^{+}$, that is, those surfaces which are good coverings over ball quotients branched along totally geodesic divisors. We first remark that these surfaces can not be ball quotients themselves, that is,

\section{Proposition 7. $\mathcal{B} \cap \mathcal{G}^{+}=\phi$.}

This is a direct consequence of Propositions 5 and 6 . In higher dimensions, it can be proved similarly that $\mathcal{B} \cap \mathcal{G}^{+}=\phi$, by comparing the ratio of the first two Chern numbers.

In the following subsections, we shall go after those known constructions of $\mathcal{B}$ in dimension two, obtained by Hirzebruch, Höfer, Livné, Kato, and Deligne-Mostow, and discuss the set $\mathcal{G}^{+}$in each case.

In higher dimensions, especially in dimension 3, the construction of Deligne and Mostow [D-M] (see also [D-M1], [M1] and [T]) also gives several compact quotients of the complex ball. However, the branching loci are no longer normal crossing, so we can not get further coverings simply by taking intersection of the corresponding subgroups. Hopefully, since the singularities of the ramification are still very mild, a modification of the argument perhaps could also yield some (quasi-) negatively curved compact Kähler manifolds, the existence of which (other than compact ball quotients) is still unknown.

\subsection{Hirzebruch-Kato surfaces: revisit.}

Let us now discuss the necessary conditions for a Hirzebruch-Kato surface to be a ball quotient. Recall that such a surface $Y$ is a good covering over $X(L)$, the blowing up of $\mathbf{P}^{2}$ at the singular multiple points $\Sigma$ of an arrangement of lines $L=\bigcup_{i=1}^{k} l_{i} .(\Sigma \neq \phi)$. The branching locus is $D=\bigcup_{i=1}^{r^{\prime}} D_{i}$ where 
the first $k$ curves are the proper transforms of $l_{i}$ and the last $r^{\prime}-k$ are part of the exceptional curves, with branching multiplicities $b=\left(b_{1}, \ldots, b_{r^{\prime}}\right)$, where $b_{i} \geq 2(1 \leq i \leq k)$ are arbitrary, and $b_{\alpha}=m_{\alpha}(b, L)$ is the least common multiple of all $b_{i}$ where $l_{i}$ passes through both the $\alpha$-th singular point $p_{\alpha}$ and at least another singular point, for each $k+1 \leq \alpha \leq r^{\prime}$. Here we wrote $\Sigma^{\prime}=\left\{p_{k+1}, \ldots, p_{r^{\prime}}\right\} \subseteq \Sigma$ for the subset where $m_{\alpha} \geq 2$. We call such a surface a Hirzebruch-Kato surface of type $L^{b}$, where by an abuse of notations, this $b$ actually means $\left(b_{1}, \ldots, b_{k}\right)$, with each $b_{i}$ greater than 1 .

Now write $f: Y \rightarrow X(L)$ for the covering map and let $R_{i}=f^{-1}\left(D_{i}\right)$. If $Y$ is a ball quotient, then all the $R_{i}, 1 \leq i \leq r^{\prime}$, will be totally geodesic curves in $Y$, so by Proposition 6 , they satisfy $\left(K_{Y}+3 R_{i}\right) R_{i}=0$. Since $R_{i}=\frac{1}{b_{i}} f^{*}\left(D_{i}\right)$, this gives

$$
\left[K_{X}+\left(1+\frac{2}{b_{i}}\right) D_{i}+\sum_{j \neq i}\left(1-\frac{1}{b_{j}}\right) D_{j}\right] D_{i}=0
$$

Also, for each $D_{i}=\overline{l_{i}}, D_{i}^{2}=1-\left|\Sigma \cap l_{i}\right|$ is a positive multiple of $R_{i}^{2}$, which is negative. So any line $l_{i}$ will have to contain at least two singular points, so $\Sigma^{\prime}=\Sigma$ and $m_{\alpha}$ is the least common multiple of $b_{i}$ for all $l_{i}$ passing through $p_{\alpha}$.

Apply this to each $D_{i}$, we get the following

Proposition 8. If a Hirzebruch-Kato surface of type $L^{b}$ is a ball quotient, then $\Sigma^{\prime}=\Sigma$, each line $l_{i}$ contains at least two points of $\Sigma$, and for each $1 \leq i \leq k, k+1 \leq \alpha \leq r$, it holds

$$
\begin{aligned}
q_{\alpha}-2 & =\frac{2}{m_{\alpha}}+\sum_{l_{i} \ni p_{\alpha}} \frac{1}{b_{i}} \\
\left|\Delta_{i}\right|-2 & =\left(\left|\Sigma_{i}\right|-1\right) \frac{2}{b_{i}}+\sum_{j \in \Delta_{i}} \frac{1}{b_{j}}+\sum_{\alpha \in \Sigma_{i}} \frac{1}{m_{\alpha}}-\left|\Sigma_{i}\right|
\end{aligned}
$$

where $p_{\alpha}$ is a $q_{\alpha}$-fold point of $L$, and $\Delta$ is the set of nodes of $L$. The subscript $i$ means taking intersection with $l_{i}$. Conversely, if both identities hold, then the Hirzebruch-Kato surface is a ball quotient.

A particularly useful consequence of the second identity is, if $\left|\Delta_{i}\right| \leq 1$, then $b_{i}>2$.

The first identity in the above is due to $[\mathrm{BHH}]$. The second identity is also known to them. 
When both equalities in Proposition 8 hold, the Hirzebruch-Kato surface $Y$ is indeed a ball quotient. This is because by adding up the first identity for all $\alpha$, one gets $\left(3 c_{2}(Y)-c_{1}^{2}(Y)\right) / d=0$ (use Proposition $5 \& 6$ once each). The ampleness of $K_{Y}$ can also be verified.

Let us now examine the algebraic possibility of the first identity in Proposition 8 . It can be written as

$$
q-2=\frac{2}{m}+\sum_{i=1}^{q} \frac{1}{b_{i}}
$$

where $q \geq 3$, each $b_{i} \geq 2$, and $m=\left[b_{1}, \ldots, b_{q}\right]$ is the least common multiple. A direct computation shows that the following are the only possibilities:

Proposition 9. The only solutions for $q$ and $b=\left(b_{1}, \ldots, b_{q}\right)$ to the above are

$$
\begin{array}{ll}
q=6: & (2,2,2,2,2,2) \\
q=4: & (3,3,3,3),(2,2,2,6),(2,2,3,3),(2,2,4,4) \\
q=3: & (5,5,5), \quad(3,6,6), \quad(3,3,9), \quad(3,4,4), \quad(3,3,5) \\
& (2,8,8),(2,5,10),(2,4,12),(2,3,18),(2,3,10)
\end{array}
$$

In other words, for a Hirzebruch-Kato surface of type $L^{b}$ to be a ball quotient, the singular points of $L$ will have to be either 3,4 , or 6 -fold points, and in those cases the multiplicities are also very restrictive. If $\left|\Delta_{i}\right| \leq 1$ for all $i$, then all $b_{i}>2$, so only 3 or 4 -fold points can occur as singular points, and with stronger restrictions on those $b_{i}$. By an easy case by case study, we get

Proposition 10. For all the line arrangements $L$ appeared in [H] and [H1], that is, simplicial real arrangements, arrangements defined by a reflection groups or cubic curves, and for any b, the only Hirzebruch-Kato surfaces that are ball quotients are the three Fermat coverings found by Hirzebruch in [H], and one more in the complete quadrilateral $A_{1}(6)$ case: $b=(3,3,3,5,5,5)$, where the three lines with $b_{i}=5$ pass through a common point.

We omit the proof here. Most of the cases can be immediately ruled out by Proposition 8 , especially the last sentence. The rest a few can be easily excluded by Proposition 9.

Although most of the Hirzebruch-Kato surfaces are not ball quotients, but their existence gives a large amount of elements in $\mathcal{G}$ over $X(L)$, therefore, by taking least common multiples as in Proposition 1, we know that 
for any $X(L)$, the set $\mathcal{G}^{+}$will be non-empty (and contains a large amount of elements) whenever $\mathcal{B}$ is not empty.

\subsection{The complete quadrilateral.}

Consider the complete quadrilateral

$$
A_{1}(6)=\left\{z_{0} z_{1} z_{2}\left(z_{0}-z_{1}\right)\left(z_{1}-z_{2}\right)\left(z_{2}-z_{0}\right)=0\right\}
$$

in $\mathbf{P}^{2}$. Denote by $X_{I}$ the blowing up of $\mathbf{P}^{2}$ at the four triple points. We use this notation because it is the first one of the three ball quotients discovered by Hirzebruch [H], we will denote the other two by $X_{I I}$ and $X_{I I I}$ later. As mentioned in $\S 1.3$, the resulting ten curves can be labeled by

$$
D=\sum_{0 \leq i<j \leq 4} D_{i j}
$$

with the property that $D_{i j} \cap D_{k l} \neq \phi \Leftrightarrow\{i, j\} \cap\{k, l\}=\phi$. When viewing $X_{I}$ as the stable compactification of the moduli space of 5-punctured $\mathbf{P}^{1}$, $D_{i j}$ is the part when the $i$-th and $j$-th punctures collide. We will fix the order of $D$ as

$$
(01,02,03,04 ; 12,13,14,23,24,34)
$$

For the "elite-eight" types of the Delight-Mostow's quotients (cf. §1.3), the corresponding type vectors $b$ are the following (we keep the same order of numbering them as in $\$ 1.3$ )

$$
\begin{array}{ll}
\text { 1. } & b^{(1)}=(5,5,5,5 ; \quad 5,5,5,5,5,5) \\
\text { 2. } & b^{(2)}=(8,8,8,8 ; \quad 4,4,4,4,4,4) \\
\text { 3. } & b^{(3)}=(3,3,3,3 ; \quad 9,9,9,9,9,9) \\
\text { 4. } & b^{(4)}=(4,4,4,4 ; \quad 6,6,6,6,6,6) \\
\text { 5. } & b^{(5)}=(12,12,6,6 ; \quad 6,4,4,4,4,3) \\
\text { 6. } & b^{(6)}=(4,12,12,12 ; 3,3,3,5,5,5) \\
\text { 7. } & b^{(7)}=(5,15,15,15 ; 3,3,3,5,5,5) \\
8 . & b^{(8)}=(8,24,24,24 ; 3,3,3,4,4,4)
\end{array}
$$

By Deligne-Mostow, all of the above eight $b$ are in $\mathcal{B}$ for $X_{I}$. The first one is also found by Hirzebruch [H], the next three by Höfer (cf. [H1] and 
[Ho]), and the seventh is exactly the Hirzebruch-Kato surface of Proposition 10. By Proposition 1, the good coverings with the same $b$ are always commensurable. This gives an abstract proof of part of the commensurability discussion of [D-M1].

Let $S_{5}$ be the symmetry group of five elements. Any $\tau \in S_{5}$ induces an order change among $D_{i j}: D_{i j} \rightarrow D_{\tau(i) \tau(j)}$. If $b$ and $b^{\prime}$ can be identified through such a reordering, certainly the corresponding coverings will be commensurable. We will denote by $\tau b$ the permutation of $b$ induces by $\tau \in S_{5}$.

Theorem 11. Any good covering of $X_{I}$ with branching locus contained in $D$ which is a ball quotients must be commensurable with one of the DeligneMostow's quotients, that is, $\mathcal{B}=\left\{\tau b^{(k)} \mid 1 \leq k \leq 8, \tau \in S_{5}\right\}$.

Proof. Suppose $b \in \mathcal{B}$ for $\left(X_{I}, D\right)$. By Proposition 6 , each curve $D_{i j}$ will give an identity

$$
1=\frac{2}{b_{i j}}+\frac{1}{b_{r s}}+\frac{1}{b_{r t}}+\frac{1}{b_{s t}}
$$

where $\{r, s, t\}=\{0,1, \ldots, 4\} \backslash\{i, j\}$. That is, the column vector

$$
y=t\left(\frac{1}{b_{01}}, \frac{1}{b_{02}}, \ldots, \frac{1}{b_{34}}\right)
$$

satisfies the system of linear equations $A y=u$, where $u={ }^{t}(1,1, \ldots, 1)$, and

$$
A=\left[\begin{array}{llllllllll}
2 & 0 & 0 & 0 & 0 & 0 & 0 & 1 & 1 & 1 \\
0 & 2 & 0 & 0 & 0 & 1 & 1 & 0 & 0 & 1 \\
0 & 0 & 2 & 0 & 1 & 0 & 1 & 0 & 1 & 0 \\
0 & 0 & 0 & 2 & 1 & 1 & 0 & 1 & 0 & 0 \\
0 & 0 & 1 & 1 & 2 & 0 & 0 & 0 & 0 & 1 \\
0 & 1 & 0 & 1 & 0 & 2 & 0 & 0 & 1 & 0 \\
0 & 1 & 1 & 0 & 0 & 0 & 2 & 1 & 0 & 0 \\
1 & 0 & 0 & 1 & 0 & 0 & 1 & 2 & 0 & 0 \\
1 & 0 & 1 & 0 & 0 & 0 & 1 & 0 & 2 & 0 \\
1 & 1 & 0 & 0 & 1 & 0 & 0 & 0 & 0 & 2
\end{array}\right]
$$

Let $\left\{e_{i j}\right\}(0 \leq i<j \leq 4)$ be the natural basis, and for $0 \leq i \leq 4$, denote by $v_{i}=\sum_{j \neq i} e_{i j}$. (Here we assume $e_{j i}=e_{i j}$ ). Then $A v_{i}=2 u$. Since $A u=5 u$, 
so for each $i, v_{i}-\frac{2}{5} u$ is in the kernel of $A$. They span a 4-dimensional space $V$

$$
V=\left\{\sum_{k=0}^{4} x_{k}\left(v_{k}-\frac{2}{5} u\right) \mid \sum x_{k}=1\right\}
$$

since the sum of all five of them is zero. On the other hand, since the upper left $6 \times 6$ corner of $A$ is non-degenerate, so the rank of $A$ is 6 , and $V$ is the kernel of $A$. Since $A\left(y-\frac{1}{5} u\right)=0$, so there exists some $x_{k}$ with $\sum x_{k}=1$ such that

$$
y-\frac{1}{5} u=\sum_{k=0}^{4} x_{k}\left(v_{k}-\frac{2}{5} u\right)
$$

That is

$$
y=\sum_{k=0}^{4} x_{k} v_{k}-\frac{1}{5} u
$$

At each position $i j$, this gives

$$
\frac{1}{b_{i j}}=x_{i}+x_{j}-\frac{1}{5}
$$

Let $\mu_{k}=\frac{3}{5}-x_{k}$, then $\sum \mu_{k}=2$, and

$$
b_{i j}=\left(1-\mu_{i}-\mu_{j}\right)^{-1}
$$

It remains to show that each $\mu_{k}$ satisfies $0<\mu_{k}<1$. Since $\mu_{i}+\mu_{j}=1-\frac{1}{b_{i j}}$ is strictly between 0 and 1 , we just need to show that each $\mu_{i}>0$. Take $\mu_{0}$ for example. By adding up the four equalities which involve $\mu_{0}$, we have

$$
3 \mu_{0}+2=4-\sum_{k=1}^{4} \frac{1}{b_{0 k}}
$$

Since each $b_{0 k} \geq 2$, we get $\mu_{0} \geq 0$. When $\mu_{0}=0$, all $b_{0 k}=2$, so $\mu_{k}=\frac{1}{2}$ for $1 \leq k \leq 4$. But then $\frac{1}{b_{12}}=1-\mu_{1}-\mu_{2}=0$, which is a contradiction. Therefore $\mu_{0}$ must be positive, and so are the other $\mu_{k}$.

In other words, we have represented these $b_{i j}$ as the reciprocal of the $1-\mu_{i}-\mu_{j}$ for five numbers in the interval $(0,1)$ whose sum is 2 . This is exactly the INT condition in [D-M]. By their result, there are exactly 27 
solutions, eight of which satisfies $\mu_{i}+\mu_{j}<1$ for all $0 \leq i<j \leq 4$, which is of course the list from 1 to 8 .

For any Hirzebruch-Kato coverings $b$, any $1 \leq k \leq 8$, and any permutation $\tau, \tau^{\prime} \in S_{5}$, the least common multiple $\left[\tau b, \tau^{\prime} b^{(k)}\right]$ are all elements in $\mathcal{G}^{+}$(assume $\tau b \neq \tau^{\prime} b^{(k)}$ ). By Theorem 4, this gives us an abundant class of examples of quasi-negatively curved surfaces.

By Proposition 5, we have

Proposition 12. If $Y$ is a good covering over $X_{I}$ with branching type $b$ along $D$, then

$$
c_{1}^{2}(Y) / c_{2}(Y)=2+\frac{1-\sum_{i=1}^{10} \frac{1}{b_{i}^{2}}}{2-\sum_{i=1}^{10} \frac{1}{b_{i}}+\frac{1}{2} \sum_{i \neq j} \frac{D_{i} D_{j}}{b_{i} b_{j}}}
$$

Note that by [Z1], for any general type compact Kähler surface with non-positive sectional curvature, the ratio $c_{1}^{2} / c_{2}$ is always between 2 and 3 , with 2 and 3 characterizing the quotients of the bidisc $D \times D$ or the ball $B^{2}$, respectively.

\subsection{Other arrangements of lines.}

Hirzebruch and Höfer also constructed compact quotient of $B^{2}$ from some other arrangement of lines. They are all homogeneous coverings (cf. $\S 1.2)$. Besides the complete quadrilateral $L_{I}=A_{1}(6)$, there are eight more arrangements involved ([H1]). We will discuss them case by case.

For an arrangement $L=\bigcup_{i=1}^{k} l_{i}$ of $k$ lines in $\mathbf{P}^{2}$, we will denote by $t_{s}$ the number of $s$-fold points, namely, the point where there are exactly $s$ lines in $L$ passing through. Again denote by $\Sigma$ the set of singular points, that is, $s$-fold points with $s \geq 3$. Let $X=X(L)$ be the blowing up of $\mathbf{P}^{2}$ at all points of $\Sigma$, and denote by $\overline{l_{i}}$ the proper transforms and $E_{\alpha}(k+1 \leq \alpha \leq r)$ the exceptional curves. Recall that a homogeneous covering of type $(n, m)$ has branching multiplicities $b_{i}=n$ along each $\overline{l_{i}}$ and $b_{\alpha}=m$ along each $E_{\alpha}$.

\section{The Hesse arrangement $L_{I I}$.}

$$
k=12, t_{2}=12, t_{4}=9 \text {, and } t_{s}=0 \text { otherwise. }
$$

It can be described as the following. Fix a smooth cubic curve $C$ in $\mathbf{P}^{2}$. Let $S$ be the set of 9 inflection points of $C$, and consider all plane cubic curves containing $S$. It forms a pencil. There are exactly four singular members of this pencil, each being the union of three lines. $L_{I I}$ is just the 
union of these 12 lines, and $\Sigma=S$ consisting of 4-fold points. By [H1], the homogeneous covering with $(n, m)=(3,3)$ or $(4,2)$ give ball quotients. (The first one is a Fermat covering, the second example of Hirzebruch's three original ball quotients).

Let us denote these 12 lines by $l_{i j}$, with $1 \leq i \leq 4$ and $1 \leq j \leq 3$, so that for each $i, L_{i}=\bigcup l_{i j}$ is a singular member in the Hesse pencil. We will denote $X\left(L_{I I}\right)$ simply by $X_{I I}$, with $D=\left(\bigcup \overline{i_{i j}}\right) \cup\left(\bigcup E_{\alpha}\right)$.

Proposition 13. For this $\left(X_{I I}, D\right)$, the only elements of $\mathcal{B}$ are those two homogeneous ones.

Proof. Suppose $\left(b_{i j}, b_{\alpha}\right)$ gives a ball quotient. Since each $l_{i j}$ contains three points of $\Sigma, \overline{l_{i j}}$ is a $(-2)$ curve. So by the local proportionality, we have

$$
3=\frac{4}{b_{i j}}+\frac{1}{b_{i k}}+\frac{1}{b_{i l}}+\sum_{p_{\alpha} \in l_{i j}} \frac{1}{b_{\alpha}}
$$

where $\{j, k, l\}=\{1,2,3\}$. Similarly, for each $\alpha$, we have

$$
2=\frac{2}{b_{\alpha}}+\sum_{l_{i j} \ni p_{\alpha}} \frac{1}{b_{i j}}
$$

Add up the first identity for the three lines in $L_{i}$, we get

$$
9=6 \sum_{j=1}^{3} \frac{1}{b_{i j}}+\sum_{\alpha=1}^{9} \frac{1}{b_{\alpha}}
$$

So the sum of $1 / b$ for the three lines in the group $L_{i}$ is the same for each $i$. Similarly, if we fix $l_{11}$ and add up the second identity for the three singular points on $l_{11}$, then all the lines in other groups appear exactly once, so we have

$$
6=2 \sum_{\alpha \in l_{11}} \frac{1}{b_{\alpha}}+\sum_{i, j} \frac{1}{b_{i j}}+\frac{2}{b_{11}}-\frac{1}{b_{12}}-\frac{1}{b_{13}}
$$

Replace the first term in the right hand side by the first identity, we know that all $b_{i j}$ are equal. All $b_{\alpha}$ are thus equal by the second identity. It's now easy to see that $(3,3)$ and $(4,2)$ are the only two possibilities.

Denote these two homogeneous type by $b^{(i)}, i=1,2$. As in the complete quadrilateral case, for any Hirzebruch-Kato covering $b$, where $b_{i j}$ could be 
arbitrary $\left(\geq 2\right.$ ), (while $b_{\alpha}$ is the least common multiple of the four $b_{i j}$ with $l_{i j}$ passing through $p_{\alpha}$ ), the least common multiple $\left[b, b^{(1)}\right]$ or $\left[b, b^{(2)}\right]$ gives elements in $\mathcal{G}^{+}$. By Theorem 4 they correspond to quasi-negatively curved surfaces.

The dual Hesse arrangement $L_{I I I}=A_{3}^{0}(3)$.

$k=9, t_{3}=12$, and $t_{s}=0$ otherwise.

This is the dual picture of the nine inflection points on a smooth plane cubic. It can be described by the equation

$$
\left(z_{0}^{3}-z_{1}^{3}\right)\left(z_{1}^{3}-z_{2}^{3}\right)\left(z_{2}^{3}-z_{0}^{3}\right)=0
$$

in the homogeneous coordinates of $\mathbf{P}^{2}$. Each line contains four triple points, so all $\overline{l_{i}}$ are $(-3)$ curves. Now suppose that a ball quotient is a good covering over $X_{I I I}$ with multiplicities $b=\left(b_{i}, b_{\alpha}\right)$ along $D$. Since $t_{2}=0$, the local proportionality principle takes the simple form:

$$
\begin{aligned}
& 2=\frac{6}{b_{i}}+\sum_{p_{\alpha} \in l_{i}} \frac{1}{b_{\alpha}} \\
& 1=\frac{2}{b_{\alpha}}+\sum_{l_{i} \ni p_{\alpha}} \frac{1}{b_{i}}
\end{aligned}
$$

Again, fix a line $l_{i}$ and add up the second identity for the four triple points on $l_{i}$, we have

$$
4=2 \sum_{p_{\alpha} \in l_{i}} \frac{1}{b_{\alpha}}+\sum_{j=1}^{9} \frac{1}{b_{j}}+\frac{3}{b_{i}}
$$

Substitute the first term on the right by the first identity, we know that all $b_{i}$ must be equal. By the second identity, all $b_{\alpha}$ must also be equal. So we are again in the homogeneous situation. The possibilities are $\left(b_{i}, b_{\alpha}\right)=(4,8)$, $(5,5),(6,4)$, or $(9,3)$, as found in [H1]. So we have

Proposition 14. For $\left(X_{I I I}, D\right)$, the only elements in $\mathcal{B}$ are the above four homogeneous coverings found by Hirzebruch and Höfer.

There are six arrangements left: $A_{3}^{0}(4), A_{3}^{0}(5)$, icosahedral, $G_{168}$, and. $A_{3}^{3}(2), A_{3}^{3}(3)$. In the first four cases, the distribution of multiple points along each line is the same, so a similar type of argument implies: 
Proposition 15. In each of four cases: $A_{3}^{0}(4), A_{3}^{0}(5)$, icosahedral, and $G_{168}$, (cf. [H1]), the only element of $\mathcal{B}$ is the (unique) homogeneous one found in [H1].

The proof is left to the readers. For the two remaining cases, namely $A_{3}^{3}(2)$ and $A_{3}^{3}(3)$, the situation is a little bit different, since in each case there are two types of lines, where the singular point distributions are not the same. It turns out that there are non-homogeneous elements in $\mathcal{B}$, that is, there are more ball quotients by Tian-Yau's theorem.

\subsection{Some new examples of 2-ball quotients.}

Let us start with the arrangement $A_{3}^{3}(m), m \geq 2$, which is defined by

$$
z_{0} z_{1} z_{2}\left(z_{0}^{m}-z_{1}^{m}\right)\left(z_{1}^{m}-z_{2}^{m}\right)\left(z_{2}^{m}-z_{0}^{m}\right)=0
$$

in the homogeneous coordinates of $\mathbf{P}^{2}$. It has the following data:

$$
k=3 m+3, \quad t_{2}=3 m, \quad t_{3}=m^{2}, \quad t_{m+2}=3
$$

Let $0 \leq i \leq 2$, and denote by $l_{i}$ the lines $z_{i}=0, \mu_{i}=l_{j} \cap l_{k}$, where $\{i, j, k\}$ is a permutation of $1,2,3$. Let $L_{i}=\bigcup_{p=1}^{m} l_{i p}$ be the group of $m$ lines passing through $\mu_{i}$. Each triple point $\alpha$ is the intersection of three lines coming from different groups, and the nodes (double points) are formed by the intersection of $l_{i}$ with each line of $L_{i}, 0 \leq i \leq 2$.

Let $X_{m}$ be the blowing up of $\mathbf{P}^{2}$ at all the $m^{2}$ triple points $\alpha$ and the three $(m+2)$-fold points $\mu_{i}$. Denote by $E_{\alpha}, E_{\mu_{i}}$ the exceptional curves, respectively. If a finite uniformization $Y$ of $\left(X_{m}, b\right)$ is a ball quotient, or equivalently, $b \in \mathcal{B}$, then we will have by local proportionality principle (Proposition 6) a bunch of equalities. It can be shown that all lines in $L_{i}$ must have equal multiplicity, which will be denoted by $c_{i}$, and all the triple points have equal multiplicity as well, denoted by $b_{\alpha}$. The equalities from the local proportionality principle now become

$$
\begin{aligned}
& m=\frac{2}{b_{\mu_{i}}}+\frac{m}{c_{i}}+\frac{1}{b_{j}}+\frac{1}{b_{k}} \\
& 1=\frac{2}{b_{\alpha}}+\frac{1}{c_{0}}+\frac{1}{c_{1}}+\frac{1}{c_{2}} \\
& m=\frac{2}{b_{i}}+\frac{m}{c_{i}}+\frac{1}{b_{\mu_{j}}}+\frac{1}{b_{\mu_{k}}} \\
& m=\frac{2 m}{c_{i}}+\frac{1}{b_{\mu_{i}}}+\frac{1}{b_{i}}+\frac{m}{b_{\alpha}}
\end{aligned}
$$


Here again $0 \leq i \leq 2$, and $\{i, j, k\}=\{0,1,2\}$.

From these equalities, we get

$$
\frac{1}{b_{i}}-\frac{1}{b_{\mu_{i}}}=a, \frac{1}{b_{i}}+\frac{m}{c_{i}}=a^{\prime}
$$

That is, they are independent of the index $i$.

Add up the first identity for $i=0,1,2$, and use the second identity, we have

$$
\sum_{i=0}^{2}\left(\frac{1}{b_{i}}+\frac{1}{b_{\mu_{i}}}\right)>m
$$

Since each $b_{i} \geq 2$, this implies

$$
\sum_{i=0}^{2} \frac{1}{b_{\mu_{i}}}>m-\frac{3}{2}
$$

So we know that $m \leq 4$.

Case 1. $m=4$

All three $b_{\mu_{i}}$ must be 1 . So all three $b_{i}$ are equal, and all three $c_{i}$ are also equal. We shall call such a situation "subhomogeneous". In this case, the equations are reduced to

$$
\begin{aligned}
& m=\frac{2}{b_{\mu}}+\frac{m}{c}+\frac{2}{b} \\
& 1=\frac{2}{b_{\alpha}}+\frac{3}{c}
\end{aligned}
$$

Here we skipped the subscript $i$. When $b_{\mu_{i}}=1$ and $m=4$, the only possibility is

$$
b_{i}=2, \quad c_{i}=4, \quad b_{\alpha}=8, \quad b_{\mu_{i}}=1
$$

Case 2. $m=3$

Since

$$
\sum_{i=0}^{2} 1 / b_{\mu_{i}}>\frac{3}{2}
$$

at least one $b_{\mu_{i}}$ will be 1 . Let us assume $b_{\mu_{0}}=1$. For $i=1,2$, by

$$
\frac{1}{b_{\mu_{0}}}+\frac{1}{b_{i}}=\frac{1}{b_{\mu_{i}}}+\frac{1}{b_{0}}
$$


we know that $b_{\mu_{i}}$ will have to be 1 since $b_{0} \geq 2$. So all three $b_{\mu_{i}}=1$ and we are again in the subhomogeneous situation. The equations reduced to

$$
\begin{aligned}
& 1=\frac{3}{c}+\frac{2}{b} \\
& 1=\frac{2}{b_{\alpha}}+\frac{3}{c}
\end{aligned}
$$

from which we get four solutions ${ }^{9}$, all are subhomogeneous:

$$
b_{\mu_{i}}=1, \quad b_{i}=b_{\alpha},\left(b_{i}, c_{i}\right) \in\{(8,4),(5,5),(4,6),(3,9)\}
$$

Case 3. $m=2$

In this case we have

$$
\sum_{i=0}^{2}\left(\frac{1}{b_{i}}+\frac{1}{b_{\mu_{i}}}\right)>2 .
$$

As in case 2 , if one of the $b_{\mu_{i}}=1$, all three of them are equal to 1 , which will violate the third identity (for $m=2$ ). So all $b_{\mu_{i}} \geq 2$.

Note that there is a symmetry between the three exceptional curves $E_{\mu_{i}}$ and the three proper transforms $\overline{l_{i}}$. That is, in we interchange $b_{i}$ with $b_{\mu_{i}}$ for all $i$, then the resulting orbifold is isomorphic to the original one. So without loss of generality, we may assume

$$
\sum_{i=0}^{2} 1 / b_{\mu_{i}}>1 .
$$

Fact. If $a \geq b \geq c \geq 2$ are three integers such that

$$
\frac{1}{a}+\frac{1}{b}+\frac{1}{c}>1
$$

Then $(a, b, c)$ is in $\{(n, 2,2),(k, 3,2)\}$ for $n \geq 2$ and $3 \leq k \leq 5$.

Using this algebraic fact, it is then straight forward to find all solutions of these equations. Let us denote by $\vec{\mu}=\left(b_{\mu_{1}}, b_{\mu_{2}}, b_{\mu_{3}}\right), \vec{b}=\left(b_{1}, b_{2}, b_{3}\right)$ and $\vec{c}=\left(c_{1}, c_{2}, c_{3}\right)$.

\footnotetext{
${ }^{9}$ Note that the position of the three exceptional curves $E_{\mu_{i}}$ and the three lines $\overline{l_{i}}$ can be interchanged, the two resulting orbifolds are isomorphic to each other, so we ignore the solutions obtained by interchanging $b_{i}$ and $b_{\mu_{i}}$ for all $i$.
} 
1). $c_{i}=4, \quad b_{\alpha}=8, \quad b_{i}=2, \quad b_{\mu_{i}}=4$;

2). $c_{i}=6, \quad b_{\alpha}=4, \quad b_{i}=2, \quad b_{\mu_{i}}=3$;

3). $b_{\alpha}=3, \vec{b}=\vec{\mu}=(2,2,3), \quad \vec{c}=(12,12,6)$;

4). $b_{\alpha}=4, \vec{b}=\vec{\mu}=(2,2,4), \quad \vec{c}=(8,8,4)$;

5). $b_{\alpha}=6, \vec{b}=\vec{\mu}=(2,2,6), \quad \vec{c}=(6,6,3)$;

6). $b_{\alpha}=6, \vec{b}=\vec{\mu}=(2,3,3), \quad \vec{c}=(6,4,4)$;

7). $b_{\alpha}=6, \vec{\mu}=(2,3,3), \quad \vec{b}=(3,3,6), \quad \vec{c}=(4,4,3)$.

Again if we interchange $\vec{b}$ and $\vec{\mu}$, we also get solutions, but the resulting orbifold remains the same. It can be verified that in each of the above cases, $\overline{c_{1}}<0$ and ${\overline{c_{1}}}^{2}=3 \overline{c_{2}}$, so by Tian-Yau's theorem, each admits a finite uniformization which is a smooth compact quotient of the complex unit ball in $\mathbf{C}^{2}$.

In each of the above cases, the combination of the ball quotient coverings with the abundant Hirzebruch-Kato coverings give a large class of elements in $\mathcal{G}^{+}$. It is easy to compute the ratio of the two Chern numbers in each case, and see their distributions.

One can also play the local proportionality principle to the other arrangements of lines listed in $[\mathrm{H}]$. It turns out that above three cases, together with the homogeneous ones and the Deligne-Mostow ones in the complete quadrilateral case, are the only combinations for ball quotients.

To start, let $X=X(L)$ be the blowing up of $\mathbf{P}^{2}$ at the singular points $\Sigma$ of $L=\bigcup_{i=1}^{k} l_{i}$. Suppose $b \in \mathcal{B}_{X, D}$, where as before

$$
D=\bigcup_{i=1}^{k} \overline{l_{i}} \bigcup_{\alpha=k+1}^{r} E_{\alpha}
$$

Let $f: Y^{2} \rightarrow X$ be a finite uniformization of $(X, b)$. Then $Y$ is a ball quotient, and the branching locus $B_{f}$ is contained in $D$ (note that we allow some $b_{i}$ to be equal to 1 ).

Let $L^{\prime}$ be all the lines $l_{i}$ in $L$ with $b_{i} \geq 2$, and $\Sigma^{\prime}$ the singular set of the subarrangement $L^{\prime}$. For any point $p_{\alpha} \in \Sigma \backslash \Sigma^{\prime}$, suppose the $q$-lines passing through $p_{\alpha}$ are $\left\{l_{i}: 1 \leq i \leq q\right\}$, with $l_{i}$ in $L^{\prime}$ for $1 \leq i \leq p$ and not in $L^{\prime}$ for $p<j \leq q$; where $p \geq 0$. Since $p_{\alpha}$ is not in $\Sigma^{\prime}$, we know that $0 \leq p \leq 2$. So the exceptional curve $E_{\alpha}=\mathbf{P}^{1}$ is an orbifold with $p \leq 2$ singular points, hence for any component $C$ of $f^{-1}\left(E_{\alpha}\right)$, the underlying space of $C$ is still a, 
rational curve, which can not be contained in a ball quotient. This shows that we must have $\Sigma^{\prime}=\Sigma$. The lines in $L \backslash L^{\prime}$ are thus redundant ones, i.e., they are in general position and do not intersect with $\Sigma^{\prime} \cap \Delta^{\prime}$, where $\Delta^{\prime}$ is the set of nodes of $L^{\prime}$. In all the arrangements appeared in [H], (except the near-pencil $A_{0}(k)$, which can be immediately ruled out), there is no redundant lines, so we may assume that for the arrangement $L$ with $b \in \mathcal{B}$ :

$$
b_{i} \geq 2 \quad \forall \overline{l_{i}}, 1 \leq i \leq k
$$

Now suppose $E_{\alpha}$ is an exceptional curve and $l_{1}, \ldots, l_{q}$ are the lines passing through $p_{\alpha}$. If $b_{\alpha} \geq 2$, then $E_{\alpha}$ is in the branching locus, and we will have

$$
q-2=\frac{2}{b_{\alpha}}+\sum_{i=1}^{q} \frac{1}{b_{i}}
$$

Since each $b_{i} \geq 2, b_{\alpha} \geq 2$, we get

$$
q-2 \leq 1+\frac{q}{2}
$$

Therefore $q \leq 6$ (and when $q=6, b_{\alpha}=b_{i}=2$ ). That is, all the singular points $p_{\alpha}$ with $b_{\alpha} \geq 2$ must be $q$-fold points with $3 \leq q \leq 6$. Another useful remark is that, as in $\S 2.4$, if a line $l_{i}$ contains less than two nodes of $L$, then $b_{i} \geq 3$. Using these observations, most of the arrangements can be ruled out quickly (for the possibility of an element of $\mathcal{B}$ ), and in the rest a few cases (e.g., $A_{1}(8), A_{1}(13)$ ), a similar type of argument as in the previous subsection will lead to the conclusion:

Proposition 16. For all the arrangements $L$ appeared in $[H]$, namely, real simplicial arrangements, and arrangements generated by unitary reflection groups or cubic curves, the only cases for $\mathcal{B}_{X(L), D}$ to be non-empty are the ones listed before, namely, the eight types for the complete quadrilateral $A_{1}(6)$, the homogeneous ones found by Höfer and Hirzebruch (Proposition 13 through 15), and the ones for $A_{3}^{3}(2)=A_{1}(9), A_{3}^{3}(3), A_{3}^{3}(4)$ listed as Cases 1 to 3 in this subsection.

Let us omit the detailed verifications here, as our main purpose in this article is to illustrate the abundance of the class $\mathcal{N}_{2}$, which, in our opinion, is already quite satisfactory by the existing elements of $\mathcal{B}$ obtained by Hirzebruch and companies.

In the next subsection, we shall discuss the situations when these further coverings over the ball quotients have smooth branching locus. These further coverings will then be negatively curved everywhere. 


\subsection{Negatively curved surfaces.}

Suppose $Y, Y^{\prime}$ are good coverings over $X$ with branching multiplicity $b$ and $b^{\prime}$ along $D$, respectively. Write $f: Y \rightarrow X$ for the covering map. Suppose $b^{\prime} \succ b$. Then by passing to an finite unbranched covering of $Y^{\prime}$ if necessary, $Y^{\prime}$ is a good covering over $Y$, and its branching locus is exactly

$$
B=f^{-1}\left(D^{\prime}\right)=f^{-1}\left(\bigcup_{b_{i}^{\prime}>b_{i}} D_{i}\right)
$$

with multiplicity $b_{i}^{\prime} / b_{i}$ along the preimage of $D_{i}$. When $Y$ is a ball quotient and $D^{\prime}$ is smooth, $Y^{\prime}$ will admit a Kähler metric which is everywhere negatively curved ([M-S] and [Z], Theorem 1). In the following, let us discuss some examples of $b \in \mathcal{B}, b^{\prime} \succ b$ with smooth $D^{\prime}=\bigcup\left\{D_{i}: b_{i}^{\prime}>b_{i}\right\}$.

Example 1. In the complete quadrilateral case. Let $1 \leq k \leq 8, \tau \in S_{5}$. and consider $b=\tau b^{(k)} \in \mathcal{B}$. Let $b^{\prime \prime}$ be the Hirzebruch-Kato covering such that the last six positions of $b^{\prime \prime}$ and $b$ are equal, and take $b^{\prime}=\left[b^{\prime \prime}, b\right]$. Then $D^{\prime}$ is contained in the the union of four disjoint curves, hence is smooth.

Example 2. Still the complete quadrilateral case. Fix the order of $D_{i j}$ as in $\$ 2.5$. Consider the seventh ball quotient

$$
b^{(7)}=(5,15,15,15 ; 3,3,3,5,5,5)
$$

This is a Hirzebruch-Kato covering itself. Consider another Hirzebruch-Kato covering

$$
b^{\prime}=(5,15,15,15 ; 15,3,3,5,5,5)
$$

In other words, by changing $b_{12}$ from 3 to 15 . The least common multiple $b_{0 i}$ remains the same for all $i$. In this case, $D^{\prime}=D_{12}$ is smooth.

Example 3. For any homogeneous $b \in \mathcal{B}$ of type $\left(b_{i}, b_{\alpha}\right)=(n, m)$, let $b^{\prime \prime}$ be the Fermat covering of order $n$ (with all $b_{i}$ and $b_{\alpha}$ equal to $n$ ), and let $b^{\prime}=\left[b^{\prime \prime}, b\right]$. Then when $m$ is not a multiple of $n, b^{\prime} \succ b$ and $D^{\prime}$ is the disjoint union of the exceptional curves, so it's smooth.

More generally, for any arrangement $L$ and any element $b \in \mathcal{B}$, let $b^{\prime \prime}$ be the Hirzebruch-Kato covering with $b_{i}^{\prime \prime}=b_{i}$ for the proper transforms of each line in $L$, and let $b^{\prime}=\left[b^{\prime \prime}, b\right]$. Most of the time, $b^{\prime} \neq b$, hence $b^{\prime} \succ b$, so $b^{\prime} \in \mathcal{G}^{+}$. The branching locus $D^{\prime}$ of the further covering is contained in the disjoint union of the exceptional curves and is thus smooth. 
Example 4. Livné ([L]) also constructed some ball quotients by cyclic coverings over level $N$ elliptic modular surfaces with branching locus the disjoint union of $N^{2}$ smooth sections. In [Hu], Hunt gives a clear description of this construction. Following the notation there $([\mathrm{Hu}], \S 4.1)$, the ball quotient $S_{3}(9)$ admits a further cover $S_{9}(9)$, while the ball quotient $S_{2}(12)$ has a further cover $S_{6}(12)$. In each case, these further coverings are also cyclic coverings along (disconnected) smooth curves. So they also provide examples of negatively curved compact Kähler surface.

Remark 1. Again it is easy to compute the ratio of the two Chern numbers for the above negatively curved surfaces. For example, in Example 2, that ratio is $437 / 151$. None of these surfaces coincide with the sequence of examples constructed in [M-S], since their Chern ratios are different. Let us omit the detailed comparisons here, it is straight-forward but lengthy.

Remark 2. Most of the surfaces constructed in this paper are only quasinegatively curved, that is, they admit Kähler metrics which are negatively curved in the complement of a finite set. It is highly unlikely that any of these surfaces can not be negatively curved, i.e., it does not admit any everywhere negatively curved Kähler metric. Although at this point we do not know how to "deform" the quasi-negatively curved metric to obtain a negatively curved one.

Let us conclude with a final remark. Let $X^{n}$ be a projective manifold and $D=\sum_{i=1}^{r} D_{i}$ a normal crossing divisor in $X$. Fix the order of $D_{i}$ and define $\mathcal{G}, \mathcal{B}$ as before. By the powerful existence result of Tian and Yau, a given integer vector $b=\left(b_{1}, \ldots, b_{r}\right)$ is in $\mathcal{B}$ or not can be verified by checking the relatively simple numerical conditions. Now if $b \in \mathcal{B}$, and let $Y$ be a finite uniformization of $(X, b), Y$ is a smooth compact quotient of the unit complex ball $B^{n}$. If $b^{\prime} \succ b$, then we can consider the normal crossing orbifold $\left(Y, b^{\prime} / b\right)$. The analytic result (Theorem 2 of [Z]) then gives a singular metric on $\left(Y, b^{\prime} / b\right)$ which is compatible with the orbifold structure. Since the sectional curvature is non-positive, it is uniformizable. In other words, if $b \in \mathcal{B}$ and $b^{\prime} \succ b$, then $\left(X, b^{\prime}\right)$ is always uniformizable by [G] and [Ha]. But it is not known whether $\left(X, b^{\prime}\right)$ is finitely uniformizable or not. (That is, $b^{\prime}$ still may not be in $\mathcal{G}$ ). In the case of arrangement of lines in dimension two, Hirzebruch-Kato coverings produces a lot of elements in $\mathcal{G}$, which, after taking the least common multiple with a $b \in \mathcal{B}$, gives us a large amount of surfaces in $\mathcal{N}_{2}$. 


\section{References.}

[BHH] G. Bathel, F. Hirzebruch and T. Höfer, Geraden-konfigurationen und Algebraishe Flächen, Vieweg, Braunschweig, 1987.

[C-Y] S-Y Cheng and S-T Yau, On the existence of a complete Kähler metric on non-compact complex manifolds and the regularity of Fefferman's equation, Comm. Pure Appl. Math. 33 (1980), 507-544.

[C-Y1] S-Y Cheng and S-T Yau, Inequality between Chern numbers of singular Kähler surfaces and characterization of orbit space of discrete group of $S U(2,1)$, Contemp. Math. 49 (1986), 31-43.

[D] M. Davis, private communications.

[D-M] P. Deligne and G.D. Mostow, Monodromy of hypergeometric functions and non-lattice integral monodromy, Publ. Math. IHES 63 (1986), 5-90.

[D-M1] P. Deligne and G.D. Mostow, Commensurabilities among lattices in $P U(1, n)$, Annals of Mathematics Studies, Princeton Univ. Press, 1993.

[F] R.H. Fox, On Fenchel's conjecture about F-groups, Mat. Tidsckrift, B, (1952), 61-65.

[F1] R.H. Fox, Covering spaces with singularities, Algebraic Geometry and Topology, Princeton Univ. Press, (1957), 243-257.

[G] M. Gromov, Hyperbolic groups, Essays in Group Theory (S. Gersten ed.), p. 75-263, Springer-Verlag, 1987.

[G-R] H. Grauert and R. Remmert, Komplexe Räume, Math. Ann. 136 (1958), 245-318.

[G-T] M. Gromov and W. Thurston, Pinching constants for hyperbolic manifolds, Invent. Math. 89 (1987), 1-12.

[H] F. Hirzebruch, Arrangements of lines and algebraic surfaces, Arithmetic and Geometry, vol II. Progress in Math, vol 36, pp. 113-140. Boston, Basel, Stuttgart: Birkhäuser 1983.

[H1] F. Hirzebruch, Algebraic surfaces with extreme Chern numbers (report on the thesis of Th. Höfer, Bonn 1984), Uspekhi Mat. Nauk 40:4 (1985), 121-129.

[Ha] A. Haefliger, Orbiespaces, Sur les Groupes Hyperboliques d'après Mikhael Gromov, Birkhäuser, Boston, 1990, 203-213.

[Ho] T. Höfer, Ballquotienten als verzweigte Überlagerungen der projektiven Ebene, Ph.D. Thesis, Bonn 1985. 
[Hu] B. Hunt, Complex manifold geography in dimension 2 and 3, J. Diff. Geom. 30 (1989), 51-153.

[K] M. Kato, On uniformization of orbifolds, Homotopy Theory and Related Topics, Kyoto (1984), Adv. Stud. Pure Math. 9 (1987), 149-172.

[K1] M. Kato, On the existence of finite principal uniformizations of $\mathbf{C P}^{2}$ along weighted line configurations, Mem. Fac. Sc. Kyushu Univ. Ser. A 38 (1984), 127-131.

[K2] M. Kato, On hyperbolic uniformization problem of normal complex reflection orbisurfaces, Topology and Computer Science, Atami (1986) Kinokuniya, Tokyo (1987), 407-427.

[K-M] S. Kudla and J. Millson, Intersection numbers of cycles on locally symmetric spaces and Fourier coefficients of holomorphic modular forms in several complex variables, Publ. Math. I.H.E.S. 71 (1990), 121-172.

[L] R. Livné, On certain covers of the universal elliptic curve, Ph.D. Thesis, Harvard Univ. 1981.

[M] G.D. Mostow, Generalized Picard lattices arising from half-integral conditions, Publ. Math. IHES 63 (1986), 91-106.

[M1] G.D. Mostow, On discontinuous action of monodromy groups on the complex $n$-ball, J. Amer. Math. Soc. 1 (1988), 555-586.

[M-S] G.D. Mostow and Y-T Siu, A compact Kähler surface of negative curvature not covered by the ball, Annals Math. 112 (1980), 321-360.

[Te] T. Terada, Probl'eme de Riemann et fonctions automorphes provenant des fonctions hyperg'eometriques de plusieurs variables, J. Math. Kyoto Univ., 13 (1973), 557-578.

[T] W. Thurston, Shapes of polyhedra, Preprint.

[T1] W. Thurston, The geometry and topology of three manifolds, Princeton Univ. (memographed notes 1978-1979).

[T-Y] G. Tian and S-T Yau, Existence of Kähler-Einstein metrics on complete Kähler manifolds and their applications to algebraic geometry, Mathematical Aspects of String Theory (S-T Yau ed.), p. 574-628, World Scientific, 1987.

[Y] S-T Yau, Calabi's conjecture and some new results in algebraic geometry, Proc. Nat. Acad. Sci. USA 74 (1977), 1798-1799.

[Z] F. Zheng, Examples of non-positively curved Kähler manifolds, Comm. Anal. Geom. 4 (1996), 129-160. 
[Z1] F. Zheng, First Pontrjagin form, rigidity and strong rigidity of nonpositively curved Kähler surfaces, Math. Zeit. 220 (1995), 159-169.

Received September 9, 1997.

DEPARTMENT OF MATHEMATICS

OHIO STATE UNIVERSITY

213 W. 18Th AVE.

Columbus, OH 43210 